\title{
Mechano-transduction to muscle protein synthesis is modulated by FAK
}

\author{
Stephan Klossner • Anne-Cecile Durieux • \\ Damien Freyssenet $\cdot$ Martin Flueck
}

Accepted: 2 March 2009 / Published online: 18 March 2009

(C) Springer-Verlag 2009

\begin{abstract}
We examined the involvement of focal adhesion kinase (FAK) in mechano-regulated signalling to protein synthesis by combining muscle-targeted transgenesis with a physiological model for un- and reloading of hindlimbs. Transfections of mouse tibialis anterior muscle with a FAK expression construct increased FAK protein 1.6-fold versus empty transfection in the contralateral leg and elevated FAK concentration at the sarcolemma. Altered activation status of phosphotransfer enzymes and downstream translation factors showed that FAK overexpression was functionally important. FAK auto-phosphorylation on Y397 was enhanced between 1 and $6 \mathrm{~h}$ of reloading and preceded the activation of p70S6K after $24 \mathrm{~h}$ of reloading. Akt and translation initiation factors $4 \mathrm{E}-\mathrm{BP} 1$ and $2 \mathrm{~A}$, which reside up- or downstream of p70S6K, respectively, showed no FAK-modulated regulation. The findings identify FAK as an upstream element of the mechano-sensory pathway of p70S6K activation whose Akt-independent regulation intervenes in control of muscle mass by mechanical stimuli in humans.
\end{abstract}

S. Klossner $\cdot$ A.-C. Durieux $\cdot$ M. Flueck

Institute of Anatomy, University of Berne, Berne, Switzerland

D. Freyssenet

Faculté de Médecine, Université Jean Monnet, Saint-Etienne, France

M. Flueck $(\bowtie)$

Institute for Biomedical Research into Human Movement and Health, Manchester Metropolitan University,

Oxford Road, Manchester M15 6BH, UK

e-mail:m.flueck@mmu.ac.uk
Keywords Focal adhesion kinase $\cdot$ S6 kinase $\cdot$ Akt . eIF4E-BP1 · Gene electrotransfer

\section{Introduction}

Striated muscle demonstrates a pronounced load-dependent phenotype (Fluck and Hoppeler 2003). This is illustrated by the dramatic reduction in mass of anti-gravitational muscles with unloading and the induction of hypertrophy with subsequent reloading. Biochemical investigations point out that modulated protein synthesis is a main mechanism underlying the early regulation in protein turnover with altered muscle loading (Rennie 2007).

Protein phosphorylation is a central theme in the regulation of protein synthesis (Chiquet and Flück 2002; Hunter 2000). Studies focusing on the molecular control of muscle size identified a number of signalling pathways that control protein synthesis via the phosphorylation of translation factors at critical amino acids, thus regulating their activity. It is known that the phospho-transfer enzymes (kinases) mTOR (mammalian target of rapamycin) and Akt are organized in a pathway that enhances translational efficiency (Bodine et al. 2001; Deldicque et al. 2008; Pallafacchina et al. 2002). This occurs via downstream activation of $70 \mathrm{kDa}$ ribosomal S6 kinase (p70S6K) and critical translation initiation factors (e.g. eIF4E-BP1, eEF2) (Gingras et al. 2001; Sandri 2008). The physiological regulation of this signalling pathway supports the view that protein synthesis is controlled by muscle activity via enhanced loading (Sandri 2008). In this respect, the activation of p70S6K correlates best with the gains in muscle mass induced by mechanical stimuli (Baar and Esser 1999; Burry et al. 2007; Nader and Esser 2001). This activation occurs when the Akt-mTOR pathway is not activated (Deldicque et al. 2008; 
Eliasson et al. 2006; Fujita et al. 2007; Terzis et al. 2008). This indicates that Akt-mTOR and p70S6K independently regulate protein synthesis. The mechano-responsive pathway which connects to p70S6K activation is not known.

The integrin-associated focal adhesion kinase (FAK) is a mechano-sensitive signalling molecule (Durieux et al. 2007), which is positioned upstream of p70S6K in cell culture (Gan et al. 2006; Malik and Parsons 1996). FAK localises to sarcolemmal focal adhesion sites, which play an important role in the conversion of mechanical stress to myocellular hypertrophy signalling (Fluck et al. 2002; Ingber 2006; Romer et al. 2006; Samarel 2005). A possible functional coupling between the activation of p70S6K and FAK is suggested by the observation that the phosphotransfer activity and phosphorylation status of both kinases correlate with protein synthesis and load-dependent increases in muscle mass (Cary and Guan 1999; Fluck et al. 2002; Ingber 2006).

We hypothesized that mechano-signalling between FAK and p70S6K is the missing molecular connection between mechanical muscle stimulation and activation of muscle protein synthesis (Baar and Esser 1999; Gan et al. 2006; Kimball et al. 2004). Towards this end, we tested whether muscle fibre-targeted FAK overexpression (Durieux et al. 2002, 2004) would enhance activation of p70S6K in tibialis anterior (TA) muscle of mice in a load-dependent manner (Gingras et al. 2001; Kimball et al. 1998; Sale et al. 1999). This was assessed in a physiological model which allows prolonged muscle unloading and subsequent reloading (Dapp et al. 2004). We were interested in elucidating the time course and relationship of the early FAK activation (Gordon et al. 2001) to the putative downstream phosphorylation of p70S6K and explored whether this pathway distinguishes to regulatory activation of p70S6K by Akt-mTOR and downstream phosphorylation of key translation factors eIF4E-BP1, eEF2 (Baar et al. 2006; Baar and Esser 1999; Gingras et al. 2001) (Fig. 1).

\section{Mechanical stress}

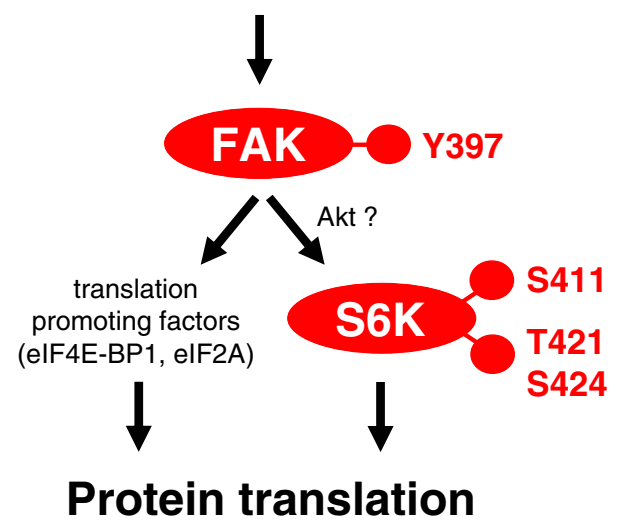

Fig. 1 Model summarizing the working hypothesis on FAK-mediated signalling towards enhanced protein translation via S6K and other translation-promoting factors

\section{Methods}

Materials

Cytomegalovirus (CMV) promoter-driven expression plasmid encoding chicken FAK gene (pCMV-FAK) or an empty plasmid (pCMV) were isolated under endotoxin-free conditions at Plasmid Factory GmbH (Bielefeld, Germany, www.plasmidfactory.de). Fluorescent-compatible mounting medium was from DAKO (DAKO, Carpinteria, CA, USA). Bicinchoninic acid assay reagents and protein A Sepharose were from Sigma (Sigma-Aldrich, St. Louis, MO, USA). The deployed antibodies against the signalling molecules in focus and the verified phosphorylation sites involved in their regulation are summarized in Table 1. Peroxidase-conjugated secondary antibodies goat anti-rabbit $\mathrm{IgG}$ and goat anti-mouse whole $\operatorname{IgG}$ were obtained from ICN Biomedicals GMBH (Germany). Alexa Fluor ${ }^{\circledR}$ 488conjugated goat-anti-rabbit IgG antibody was from Molecular probes (Invitrogen Ltd, Paisley, UK).

Super Signal West Femto Kit and Kodak XAR5 films were from Pierce (Perbio Science, Lausanne, Switzerland) and Sigma (Buchs, Switzerland), respectively.

Animals

The animal protocol was approved by the Animal Protection Commission of the Canton Berne, Switzerland. The 6-month-old male mice 129/SVEV weighed $35.4 \pm 0.7 \mathrm{~g}$ $(n=17)$ before the intervention. They were housed individually in a temperature-controlled room $\left(21^{\circ} \mathrm{C}\right)$ with a 12:12 h light-dark cycle. Animals were allowed food and water ad libitum.

Gene electro transfer

Intramuscular gene transfer was achieved via injection of plasmid DNA and subsequent electric pulse delivery in both legs basically as previously described (Durieux et al. 2002; Durieux et al. 2004; Pallafacchina et al. 2002). The mice were individually anesthetized with isoflurane and the lower limbs were shaved. Twenty-five microgram of expression plasmid in $25 \mu$ physiological saline solution $(0.9 \% \mathrm{NaCl})$ was injected with a sterile $100 \mu \mathrm{l}$ syringe into the tibialis anterior (TA) muscle. After 5 min of incubation, electric pulses ( 2 trains of 100 pulses of $100 \mu$ s each at $50 \mathrm{~mA}$ ) were delivered at four different locations in the belly portion using the GET42 pulser with needle electrodes (E.I.P. Electronique et Informatique du Pilat, Jonzieux, France). This technique allows to maintain the overexpression for more than 1 week (Fluck et al. 2008). Typically, mice recovered rapidly from this procedure and began to move freely $2 \mathrm{~h}$ after the intervention. 
Table 1 Name, function, epitope and source of all primary antibodies used to identify FAK-dependent signalling in this study

\begin{tabular}{|c|c|c|c|}
\hline Antibody & Function & Epitope & Source \\
\hline FAK & Tyrosine kinase & N-terminal (A-17) & Santa Cruz, CA, USA \\
\hline FAK & Tyrosine kinase & C-terminal (Lulu) & $\begin{array}{l}\text { Gift from A. Ziemiecki } \\
\text { (Flück et al. 1999) }\end{array}$ \\
\hline pFAK-Y397 & $\begin{array}{l}\text { Major auto-phosphorylation } \\
\text { and activation site }\end{array}$ & Phospho-Tyrosine 397 (Y397) & Santa Cruz, CA, USA \\
\hline pFAK-Y397 & $\begin{array}{l}\text { Major auto-phosphorylation } \\
\text { and activation site }\end{array}$ & Phospho-Tyrosine 397 (Y397) & $\begin{array}{l}\text { BioSource Europe } \\
\text { (Nivelles, Belgium) }\end{array}$ \\
\hline p70S6K & Key regulator of mRNA translation & p70S6K (C-18) & Santa Cruz, CA, USA \\
\hline p-p70S6K-S411 & Pre-activation of p70S6K & Phospho-Serine 411 (S411) & Santa Cruz, CA, USA \\
\hline p-p70S6K-T421/S424 & $\begin{array}{l}\text { Pre-activation of p70S6K } \\
\quad \text { (a Serine/Threonine kinase) }\end{array}$ & $\begin{array}{l}\text { Phospho-Threonine } 421 \\
\quad \text { and Serine } 424(\mathrm{~T} 421 / \mathrm{S} 424)\end{array}$ & Santa Cruz, CA, USA \\
\hline p-eIF2alpha-S52 & Regulation of translation initiation & Phospho-Serine 52 (S52) & $\begin{array}{l}\text { BioSource Europe, } \\
\text { Nivelles, Belgium }\end{array}$ \\
\hline p-4E-BP1-T37/46 & Regulation of translation initiation & Phospho-Threonine 37 and 46 (T37/46) & $\begin{array}{l}\text { Cell Signaling Technology, } \\
\text { Danvers, MA, USA }\end{array}$ \\
\hline p-Akt-T308 & Activation of signal transduction & Phospho-Threonine 308 (T308) & Santa Cruz, CA, USA \\
\hline p-Akt-S473 & Activation of signal transduction & Phospho-Serine 473 (S473) & $\begin{array}{l}\text { Cell Signaling Technology, } \\
\text { Danvers, MA, USA }\end{array}$ \\
\hline p-ERK & Activation of signal transduction & Phospho-Threonine 204 (T204) & Santa Cruz, CA, USA \\
\hline
\end{tabular}

\section{Modulation of muscle loading}

Animals were subjected to either of five different loading conditions basically as previously described (Dapp et al. 2004; Fluck et al. 2005). Two days after the gene electrotransfer, animals were subjected to 7 days of hindlimb unloading (HU) by tail suspension or kept as cage controls (CC). Subsequently, a set of suspended animals was subjected to reloading for $1 \mathrm{~h}$ (R1), $6 \mathrm{~h}$ (R6) or $24 \mathrm{~h}$ (R24). Cage activity was encouraged in the first hour of reloading by tipping the finger into the suspension cage. TA muscles were harvested from anesthetized animals, rapidly weighed, frozen in nitrogen-cooled isopentane and stored at $-70^{\circ} \mathrm{C}$ for subsequent analysis.

\section{Confocal microscopy}

The subcellular localization of FAK was detected on cryosections as previously described (Flück et al. 1999; Fluck et al. 2002), but with the modification that fluorescentlabelled secondary antibodies were used. The deployed primary FAK antibody A-17 was applied at a 1:100 dilution in 0.3\% BSA/PBS, reacted with 200-fold diluted Alexa488conjugated anti-rabbit IgG and embedded in fluorescentcompatible mounting medium. Fluorescence and digital phase contrasts were analyzed with a Leica TCS SP5 confocal microscope (Leica Microsystem CMS, Mylton Keynes, UK). The subcellular localization of FAK was analyzed on cryosections of cage control, of $6 \mathrm{~h}$ and of $24 \mathrm{~h}$ reloaded animals combined together. Sarcolemmal and sarcoplasmic FAK staining was quantified with the colocalization macro of Leica Application Suite. Signals were calculated from the mean of integrated channel intensity of the sarcoplasmic and sarcolemmal region of each fibre along six parallel "optical slices" at $10 \mu \mathrm{m}$ distance. Six fibres were quantified per treatment. Raw data were exported for statistical analysis.

\section{Immunoblotting}

SDS-PAGE, western blotting and immunodetection were performed with specific antibodies (Table 1) as previously described (Flück et al. 1999; Gordon et al. 2001). Standardized amounts of protein $(20 \mu \mathrm{g})$ were loaded per well. Signal was recorded with enhanced chemiluminescence by using the Super Signal West Femto Kit and Kodak XAR5 films. The signal intensity of the specific protein band was determined using the line and band density mode in the Quantity One 1-D analysis software 4.6.1 (Bio-Rad, Life Science Research, Hercules, CA, USA).

FAK phosphorylation on Y397 was determined after immunoprecipitation. One milligram of soluble protein in $750 \mu \mathrm{l}$ RIPA buffer was isolated and combined with $1 \mu \mathrm{l}$ pFAK serum from BioSource and $10 \mu \mathrm{l}$-FAK from Santa Cruz. Five milligram Protein A Sepharose (Sigma) was added and incubated with shaking at $4^{\circ} \mathrm{C}$ for $2 \mathrm{~h}$. After incubation of antibodies with the protein sample over night, the immunocomplexes were precipitated by centrifugation 
for $10 \mathrm{~min}\left(10,000 \mathrm{~g}, 4^{\circ} \mathrm{C}\right)$, washed twice in $1 \mathrm{ml}$ RIPA and resuspended in SDS loading buffer for separation by $7.5 \%$ SDS-PAGE. Proteins were subsequently subjected to immunoblotting for FAK protein with antibody "Lulu" (Table 1).

In vitro S6 kinase activity assay

Phosphotransfer activity of p70S6K was evaluated in vitro. S6 kinase phosphorylation was initiated by the addition of $75 \mu \mathrm{g}$ protein homogenate to $45 \mu \mathrm{l}$ preheated phosphorylation mixture including S6K substrate peptide (RRRLRRLRA) at $30^{\circ} \mathrm{C}$ basically as described (Akimoto et al. 1998; Napoli et al. 1998). The reaction was stopped after 5 min by spotting $20 \mu \mathrm{l}$ on a Whatman P81 filter and by washing in $75 \mathrm{mM} \mathrm{H}_{3} \mathrm{PO}_{4}$ and acetone (Fluck et al. 2000). Quantification of incorporated 32P was performed by liquid scintillation counting. Two technical replicas were measured from each sample. Background values (radioactivity background: same reaction without homogenate; homogenate background: same reaction without substrate peptide) were subtracted.

\section{Statistics}

For immunoblotting, samples from contralateral muscle pairs (i.e. pCMV-FAK and pCMV-transfected TA) were separated in adjacent lanes of the SDS-PAGE gel and a reference sample was run in all gels. Data were related to the reference sample. Statistical analysis was carried out with Statistica 6.1 (StatSoft Europe, Hamburg, Germany). The expression and phosphorylation level in pCMV-transfected muscles, as well as body and muscle weight, were evaluated with a one-factor analysis of variance (ANOVA). Differences between pCMV-transfected and pCMV-FAK muscle pairs were analyzed with a Friedman-ANOVA with repeated measurements. Subsequently, a Fisher post hoc test was carried out to localize the effect. Linear regression analysis was carried out with Pearson correlation. A $p$ value of 0.05 was selected as the significance level for all tests. Values are given as means \pm standard error (SE).

\section{Results}

Interaction of electrotransfer and muscle loading

TA muscle pairs of adult mice were subjected to gene electrotransfer. The right TA muscle was transfected with constitutive-active plasmid for chicken FAK, i.e. pCMV-FAK. The contralateral TA was transfected with an empty pCMV plasmid. Subsequently, animals were subjected to the different loading protocols. 7 days of unloading produced the expected drop in body weight, which did not recover within $24 \mathrm{~h}$ of reloading (Table 2). The ratio of the TA muscle to the body weight was unchanged and not affected by the unloading and reloading for both transfections.

\section{FAK overexpression}

The expression level and localization of FAK protein in transfected TA muscle was analyzed by immunoblotting and immunofluorescence. Figure $2 \mathrm{a}$ and $\mathrm{b}$ depict FAKimmunolocalization after pCMV-FAK transfection in a single positive fibre. Figure $2 \mathrm{c}$ shows the mosaic pattern of FAK localisation after pCMV-FAK transfection at a lower magnification. The amount of FAK localized in the sarcolemma was threefold higher than the amount of FAK in the sarcoplasm, regardless of the muscle loading (Fig. 2d). A proportion of fibres in empty-transfected muscle demonstrated FAK-immunostaining, but staining intensity was substantially more pronounced in pCMV-FAK transfected muscle (data not shown). Gene electrotransfer also caused muscle fibre damage in the transfected region, which was comparable for both conditions of transfection (data not shown). Qualitative immunoblotting identified a 1.6-fold increase in FAK protein levels in cage controls 9 days after electrotransfer (Fig. 3a). The FAK protein was detected as a single band at $125 \mathrm{kDa}$ in empty and chicken FAK transfected mouse TA muscles (Fig. 3b).

Effect of muscle loading on FAK overexpression and activity

The difference in FAK expression was load-dependent. FAK overexpression in pCMV-FAK transfected muscle

Table 2 Alterations in body weight and tibialis anterior (TA) muscleto-body weight ratio with the intervention

\begin{tabular}{llllll}
\hline & HU7 & R1 & R6 & R24 & CC \\
\hline $\begin{array}{l}\text { Body weight versus pre } \\
\text { intervention (\%) }\end{array}$ & -14 & -15 & -11 & -12 & $-3^{*}$ \\
$\begin{array}{l}\text { SE (\%) } \\
\text { TA/body weight pCMV }\end{array}$ & 3 & 2 & 4 & 1 & 2 \\
$\quad[\mathrm{mg} / \mathrm{g}]$ \\
$\begin{array}{l}\text { SE } \\
\begin{array}{l}\text { TA/body weight pCMV-FAK } \\
\quad(\mathrm{mg} / \mathrm{g})\end{array}\end{array}$ & 1.11 & 1.29 & 1.19 & 1.17 & 1.21 \\
SE & 0.09 & 0.05 & 0.01 & 0.17 & 0.07 \\
\hline
\end{tabular}

Effect of time of reloading on the body weight was analyzed with a one-factor ANOVA, the effect of time of reloading and transfection on TA-to-body weight on contralateral muscle pairs was verified with an ANOVA for repeated measures. The effect was localized with a FisherLSD post hoc test $(*)$. Body weight was significantly higher in cage control (CC) animals than at all other time points (7 days of unloading and $0,1,6$ and $24 \mathrm{~h}$ of reloading) 
Fig. 2 FAK staining in muscle cross-sections. a, b Representation of a FAK-positive fibre in a cross-section from a TA muscle transfected with pCMV-FAK plasmid without (a) and with differential interference contrast (b). c Lower magnification picture of FAK immunostaining after pCMV-FAK plasmid transfection. The majority of exogenous FAK protein within the FAK positive fibres was located near the plasma membrane (sarcolemma). Bar denotes $50 \mu \mathrm{m}$. d Mean $\pm \mathrm{SE}$ of FAK abundance per fibre (\% of total pixel count) associated with the sarcolemma and the sarcoplasma
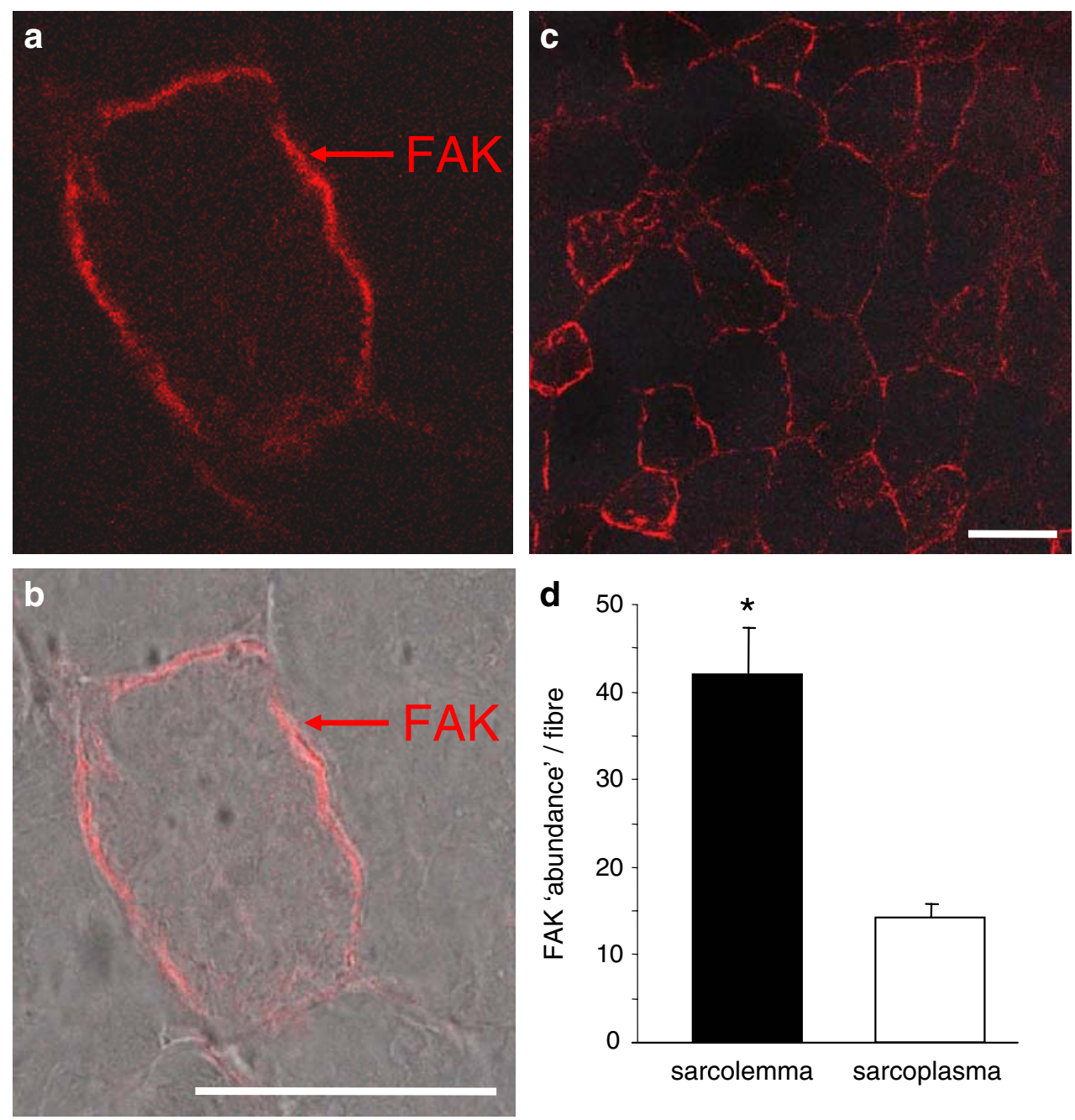

was lost with 7 days of unloading but was re-established within $6 \mathrm{~h}$ of reloading (Fig. 3a). This increase in FAK protein with reloading was preceded by enhanced phosphorylation of FAK on the major activation site Y397 after $1 \mathrm{~h}$ of reloading (Fig. 3c, d). This tyrosine phosphorylation with reloading was transient and lost after $24 \mathrm{~h}$ of reloading.

\section{Load-dependent p70S6K-signalling}

We tested whether the added mechanical stress of reloading in combination with FAK-overexpression would activate p70S6K in muscle deconditioned by unloading. Unloading did not bring about significant differences in p70S6K amount, phosphorylation and phosphotransfer activity between pCMV-FAK- and pCMV transfected TA muscles (Fig. 4a-d). Subsequent reloading altered the phosphorylation status of p70S6K in deconditioned TA muscle both qualitatively and quantitatively: $6 \mathrm{~h}$ after the first ground contact of hindlimbs, p70S6K was increasingly phosphorylated on the dual site T421/S424 in pCMV-FAK transfected muscle versus their contralateral controls. This response peaked after $24 \mathrm{~h}$ of reloading (Fig. $4 \mathrm{a}, \mathrm{b}$ ). Phosphorylation on S411 showed a near trend for FAK-transfection mediated elevation $6 \mathrm{~h}$ after reloading $(p=0.10)$. Functionally important regulation of p70S6K by loading was emphasized by a significant 3.8-fold enhanced p70S6K-phosphotransfer activity in pCMV-FAK- versus pCMV transfected muscle after $24 \mathrm{~h}$ of reloading (Fig. 4c). p70S6K protein levels were not affected by reloading between FAK overexpressing and contralateral control muscle (Fig. 4d). p70S6K phosphorylation status of the verified sites pS411 and pT421/S424 and phosphotransfer activity of p70S6K during the reloading response were significantly correlated for both transfection conditions (i.e. pS411: $r=0.73$; pT421/S424: $r=0.60$ ).

To test whether this activation of p70S6K was induced by an activation of Akt, we analyzed the phosphorylation status of Akt on its major phosphorylation sites T308 (Fig. 5a) and S473 (Fig. 5b). No activation of Akt was detected throughout the intervention. 

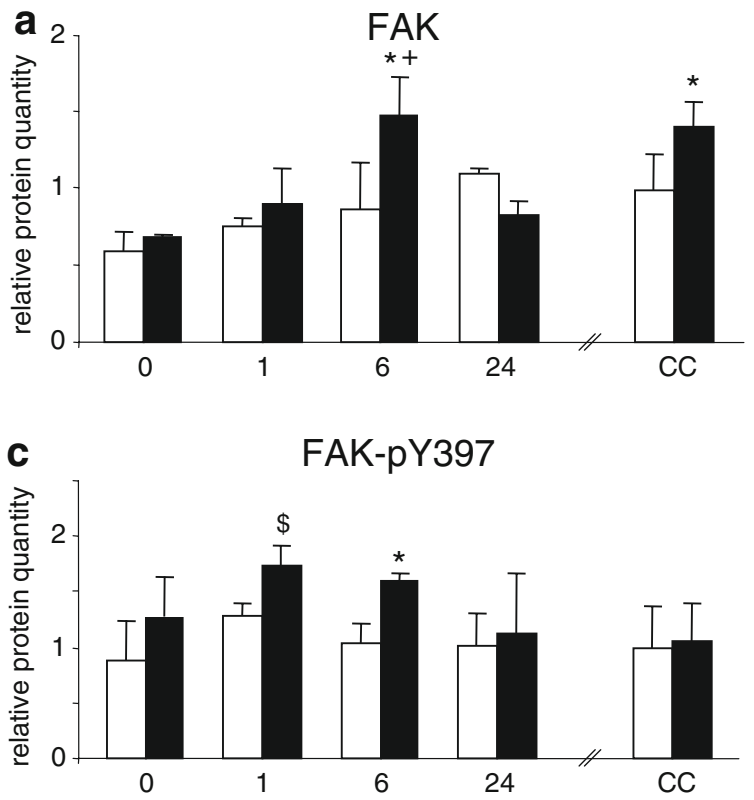

Fig. 3 Assembly showing FAK protein and tyrosine 397 phosphorylation levels in mouse TA muscle in function of gene transfer and hindlimb un- and reloading. a Mean \pm SE of FAK signal relative to pCMV-empty transfected CC value in muscles from the different loading interventions: white bars pCMV-empty plasmid in left TA muscle; black bars pCMV-FAK plasmid in TA right muscle. CC cage control, 9 days after transfection; $0: 7$ days of hindlimb unloading and no reloading; $1: 1 \mathrm{~h}$ of reloading; $6: 6 \mathrm{~h}$ of reloading, $24: 24 \mathrm{~h}$ of reloading. b Representative immunoblot showing FAK in cage control muscle

FAK-signalling to translation factors

The verification of two key translation initiation factors identified no significant effect of FAK-transfection. Neither phosphorylation on the key regulatory sites T37/T46 in the eukaryotic translation initiation factor $4 \mathrm{E}$ binding protein 1 (eIF4E-BP1) nor the activating site S52 in the eukaryotic initiation factor 2 alpha (eIF2A) were significantly affected by the introduction of FAK (Fig. 6a, b). Reloading per se increased; however, S52 phosphorylation of eIF2A after one hour of reloading in both transfection conditions. The total protein content of eIF2 was unchanged (data not shown). eIF2A pS52 was negatively correlated to p70S6K pT421/S424 and eIF4E-BP1 pT37/T46 phosphorylation.

\section{Discussion}

Mechano-transduction: from FAK to an activation of protein synthesis

Our molecular analysis demonstrates that the experimental enhancement of FAK-signalling transduces a mechanically imposed stimulus to the delayed activation of p70S6K-

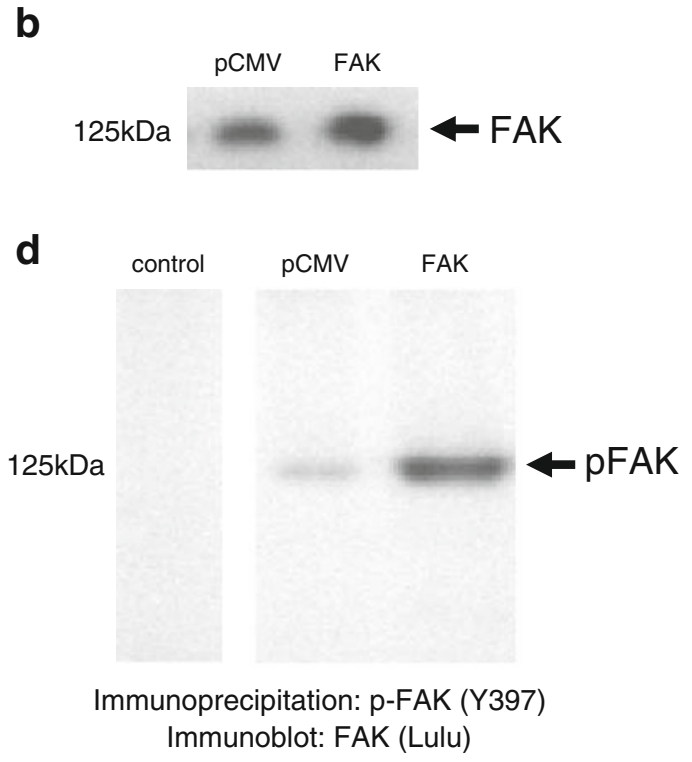

9 days after transfection. c Mean \pm SE of FAK relative to pCMVempty transfected CC value of FAK phosphorylation on Y397 in cage controls and with un- and reloading. d Representative immunoblot detecting FAK phosphorylation on Y397 in immunoprecipitates in function of gene transfer and hindlimb un- and reloading. Note the absence of FAK protein in the negative control reaction where no antibody was added to the precipitation. Friedman-ANOVA: $+p<0.05$ versus time point $0 ; * p<0.05$ versus contralateral control; $\$ 0.05<$ $p<0.10$ versus contralateral control

signalling. The temporal relationships between FAK and p70S6K-phosphorylation and phosphotransfer activity establish that the load-modulated signalling pathway of translation control in muscle is modulated by FAK. The time-course and specificity of p70S6K phosphorylation allows important regulatory conclusions on the pathway connecting FAK to p70S6K activation. The FAK-modulated phosphorylation on S411 and the dual phosphorylation T421 and S424 of p70S6K points to the involvement of serine/threonine kinases since FAK activity explicitly targets tyrosine residues. The measured p70S6K phosphorylation sites are targeted by numerous kinases, including PI3K (Dardevet et al. 1996; Thomas and Hall 1997), Akt (Hemmings 1997), PDK1 (Chan et al. 1999), mTOR (Isotani et al. 1999) and PKC (Laser et al. 1998), which could mediate the identified connections of FAK and p70S6K activation in vivo (Baar et al. 2006; Baar and Esser 1999; Gan et al. 2006).

In this context we further tested differences in phosphorylation of the serine/threonine-protein kinases Akt at T308 and S473 (Fig. 5), as wells as extracellular signalregulated kinase (ERK) at T204 (results not shown), without being able to detect any changes neither in the FAK overexpression nor in the empty transfected muscles. These observations support the view that an activation of 

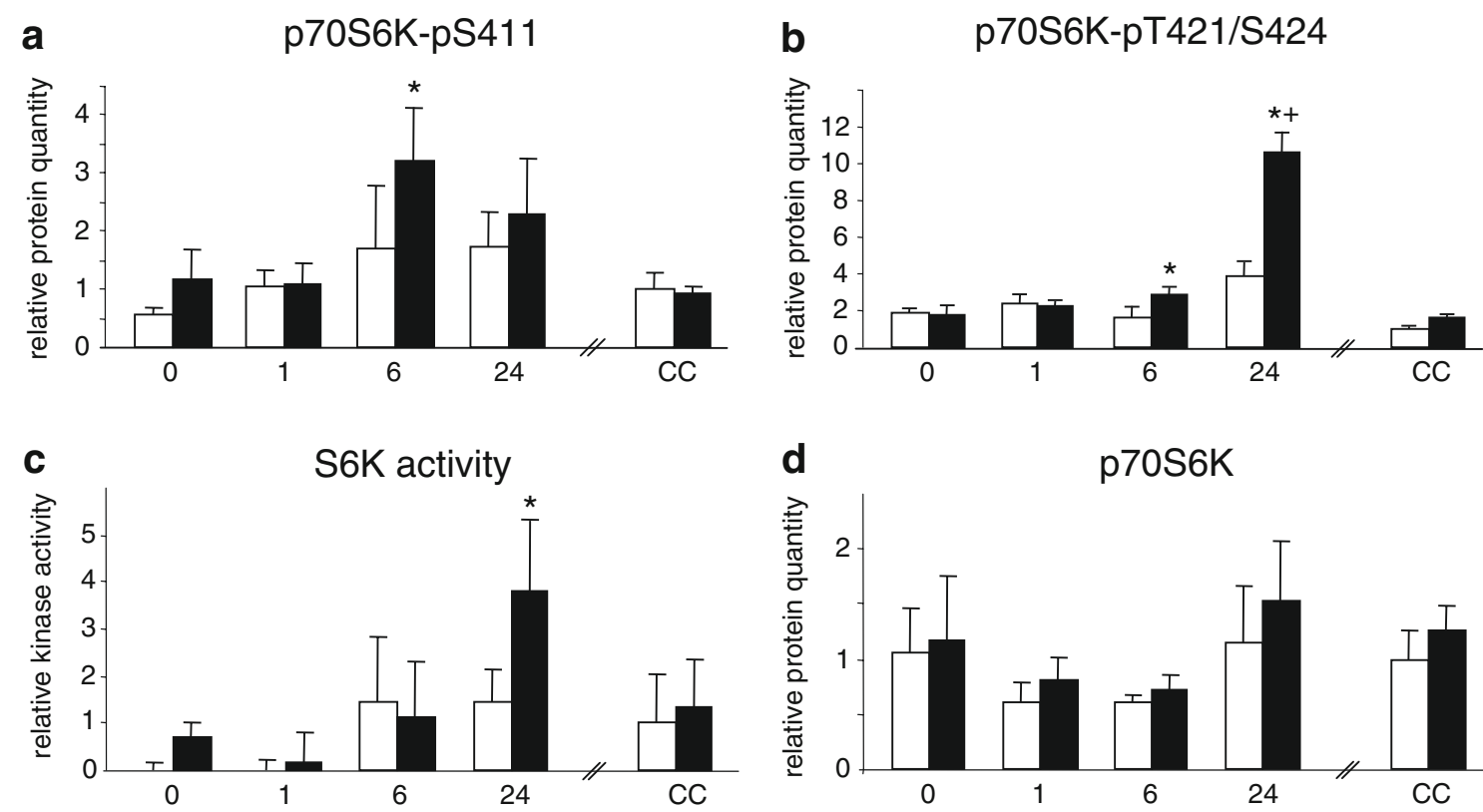

\section{S6K activity}

e

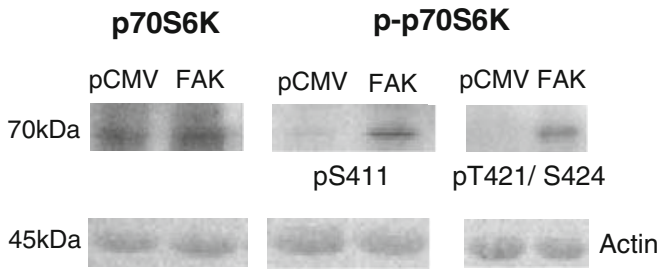

Fig. 4 Effect of FAK overexpression on p70S6K phosphorylation on S411 (a) and on T421/S424 (b), S6K in vitro kinase activity (c) and p70S6K protein level (d). Data are presented relative to the pCMVempty transfected CC value. White and black bars denote mean $\pm \mathrm{SE}$

Akt (and possibly mTOR) primarily responds to insulinand feeding-dependent signals to muscle mass. It seems that Akt and mTOR are resistive to mechanical stimuli in the fasted state (Deldicque et al. 2008; Sandri 2008). Rather, our results confirm the transient downregulation of Akt activation state shortly after a mechanical stimulus (Fig. 5b; Deldicque et al. 2008). The activation of p70S6K to mechanical stimulation in humans is also apparent when the activation status of Akt or mTOR is not altered (Deldicque et al. 2008; Eliasson et al. 2006; Fujita et al. 2007; Terzis et al. 2008). These observations and our results suggest that p70S6K activation by mechanical signals is Akt-independent.

The observations recapitulate the reported role of p70S6K phosphorylation for protein synthesis in intact muscle. For instance, p70S6K phosphorylation on T421 and S424 has been shown to correlate with gains in muscle mass in different animal models (i.e. stretch and resistance exercise) for muscle hypertrophy (Baar and Esser 1999; Bodine et al. 2001; Reynolds et al. 2002; Spangenburg

of signal in pCMV-transfected and pCMV-FAK-transfected muscle, respectively. Friedman-ANOVA: $* p<0.05$ versus contralateral control; $+p<0.05$ time point 0 . e Representative panels visualizing the quantified p70S6K signals in western blots as shown in a-d

et al. 2008; Thomson and Gordon 2006). The assessed sites control biochemical function of p70S6K and their enhanced phosphorylation is believed to stimulate protein synthesis in vitro (Gingras et al. 2001; Kimball et al. 1998; Sale et al. 1999). Phosphorylation of p70S6K on S411 and the tandem T421/S424 relieves the phospho-transfer activity from autoinhibition prior to a full activation of the enzyme (Pullen and Thomas 1997). The correlation of phosphorylation at the latter tandem sites in FAK-transfected muscles supports the notion of a functional implication of FAKstimulated p70S6K activation in translation control in vivo.

The findings also corroborate earlier suggestions on a role of FAK in protein synthesis (Baar and Esser 1999; Kimball et al. 2004) and cell size regulation (Ingber 2006). Our present results now imply a functional contribution of FAK in modulating the load-induced hypertrophy response of muscle due to p70S6K-mediated induction of protein translation. They therefore expand the recently published summarizing scheme about signalling during muscle hypertrophy (Sandri 2008). 


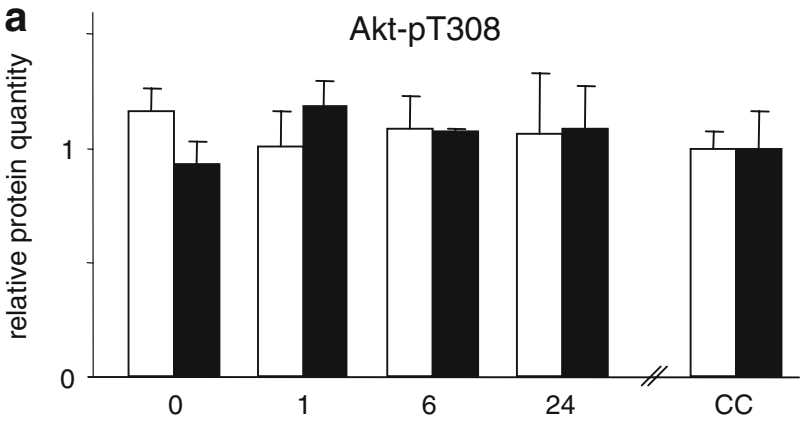

b

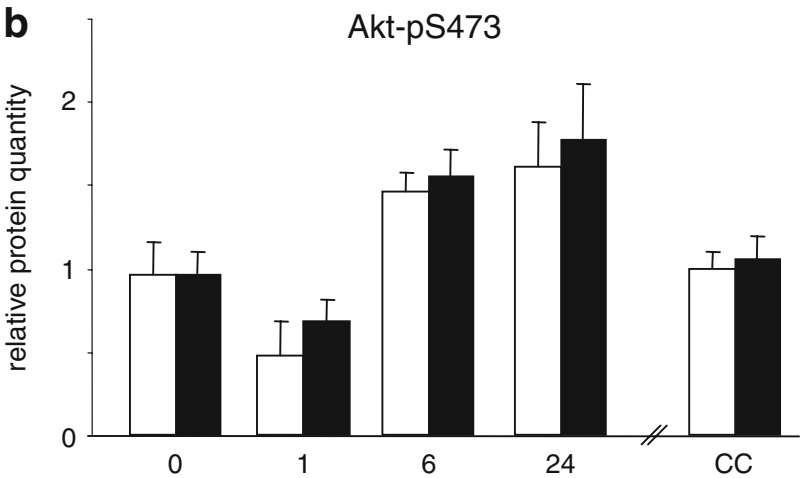

C

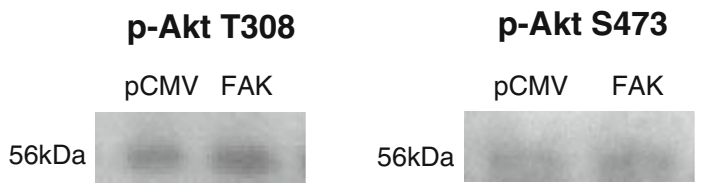

Fig. 5 Effect of FAK overexpression on Akt phosphorylation on T308 (a) and S473 (b). Representative detection of the protein in immunoblots of phosphorylated Akt at T308 and S473 are shown in (c). Data are presented relative to the pCMV-empty transfected CC value. White and black bars denote mean $\pm \mathrm{SE}$ of signal in pCMVtransfected and pCMV-FAK-transfected muscle, respectively

\section{Study limitations}

Technical considerations on the deployed experimental approach indicate the important contribution of biological variables other than FAK and muscle loading to the measured FAK-mediated signalling. Foremost this is presented by the reported damage response of transfected muscle portions by the selected methodology of gene transfer (Durieux et al. 2002, 2004; Gehl et al. 1999). In our setting, this bias was controlled by comparing the net effect of transfection in contralateral muscle pairs between FAKproducing and empty expression plasmid. This paired design allowed the identification of statistically significant effects of FAK-transfection on p70S6K-signalling in transfected TA muscles which were mechano-modulated throughout time. This is considerable taking into account the incomplete percentage of muscle fibre transfection (Durieux et al. 2002, 2004), the moderate responsiveness of
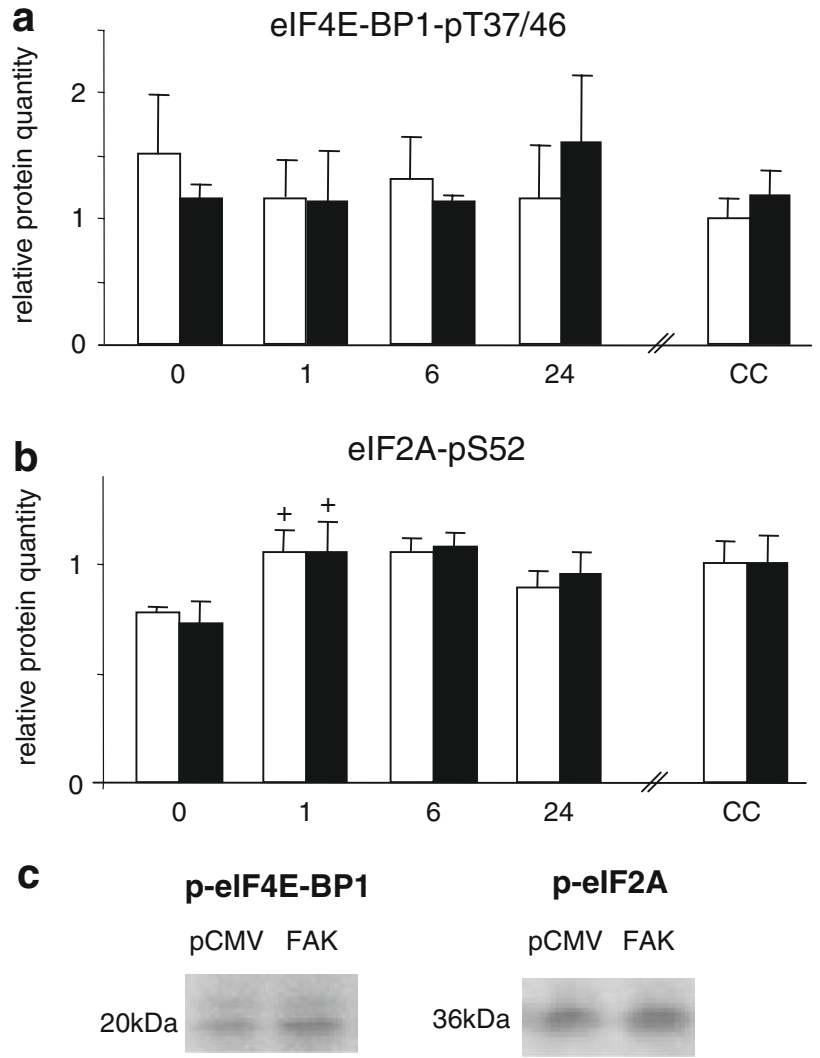

Fig. 6 Effect of FAK overexpression on eIF4E-BP1 phosphorylation on T37/46 (a) and eIF2A on S52 (b). Data are presented relative to the pCMV-empty transfected $\mathrm{CC}$ value. White and black bars denote mean \pm SE of signal in pCMV-transfected and pCMV-FAK-transfected muscle, respectively. Friedman-ANOVA: $+p<0.05$ versus time point 0 . $\mathbf{c}$ shows representative detection of proteins in immunoblots

TA muscle to hindlimb unloading compared to other leg muscle groups (Carlson et al. 1999; Dapp et al. 2004) and the restrictions imposed by the relatively low number of animals per experimental group. This highlights the resolution power of our approach for exposing muscle signalling.

Specificity of FAK-mediated mechano-signalling

The measured control of FAK protein and tyrosine phosphorylation levels in transfected muscles implies an important physiological modulation of FAK function by muscle loading. This regulation of Y397 phosphorylation and the amount of FAK between pCMV-FAK- and pCMV transfected muscle differed with regard to the "effective" time of loading. For instance, total level of Y397 phosphorylation was transiently enhanced by reloading without a change in FAK protein. In cage controls, no difference in Y397 phosphorylation was, however, between FAK-transfected and empty-transfected TA muscle when total FAK protein levels were elevated. We suggest that the elevated FAK activation within the first hours of reloading reflects the possibly 
higher mechanical impact of normal cage activity on mechano-transduction in TA muscle after a period of unloading.

\section{Conclusion}

Our investigation on the mechano-biology of striated muscle identifies FAK as an upstream element of the mechanoregulated pathway for activation of ribosomal S6 kinase. The disconnection of this pathway towards protein translation from the commonly invoked Akt-mTOR signal has major bearing for control of muscle mass in physiological situations.

Acknowledgments Special thanks are addressed to the personal of the University of Berne: PhD Sonja Mund and David Haberthür for their work in breeding and housing the animals. We also acknowledge the assistance of PhD Michael Altmann from the Institute for Biochemistry and Molecular Biology and PhD Jürg Reichen from the Department of Clinical Pharmacology and PhD Oliver Baum from the Institute of Anatomy for their technical advices. This study was supported by the Swiss National Science Foundation and a start up grant from the Manchester Metropolitan University.

\section{References}

Akimoto K, Nakaya M, Yamanaka T, Tanaka J, Matsuda S, Weng QP, Avruch J, Ohno S (1998) Atypical protein kinase Clambda binds and regulates p70 S6 kinase. Biochem J 335(Pt 2):417-424

Baar K, Esser K (1999) Phosphorylation of p70(S6k) correlates with increased skeletal muscle mass following resistance exercise. Am J Physiol 276:C120-C127

Baar K, Nader G, Bodine S (2006) Resistance exercise, muscle loading/unloading and the control of muscle mass. Essays Biochem 42:61-74

Bodine SC, Stitt TN, Gonzalez M, Kline WO, Stover GL, Bauerlein R, Zlotchenko E, Scrimgeour A, Lawrence JC, Glass DJ, Yancopoulos GD (2001) Akt/mTOR pathway is a crucial regulator of skeletal muscle hypertrophy and can prevent muscle atrophy in vivo. Nat Cell Biol 3:1014-1019

Burry M, Hawkins D, Spangenburg EE (2007) Lengthening contractions differentially affect $\mathrm{p} 70(\mathrm{~s} 6 \mathrm{k})$ phosphorylation compared to isometric contractions in rat skeletal muscle. Eur J Appl Physiol 100:409-415

Carlson CJ, Booth FW, Gordon SE (1999) Skeletal muscle myostatin mRNA expression is fiber-type specific and increases during hindlimb unloading. Am J Physiol 277:R601-R606

Cary LA, Guan JL (1999) Focal adhesion kinase in integrin-mediated signaling. Front Biosci 4:D102-D113

Chan TO, Rittenhouse SE, Tsichlis PN (1999) AKT/PKB and other D3 phosphoinositide-regulated kinases: kinase activation by phosphoinositide-dependent phosphorylation. Annu Rev Biochem 68:965-1014

Chiquet M, Flück M (2002) Early responses to mechanical stress: from signals at the cell surface to altered gene expression. In: Storey KB, Storey JM (eds) Protein adaptations and signal transduction. Elsevier Science, BV, pp 97-109

Dapp C, Schmutz S, Hoppeler H, Fluck M (2004) Transcriptional reprogramming and ultrastructure during atrophy and recovery of mouse soleus muscle. Physiol Genomics 20:97-107
Dardevet D, Sornet C, Vary T, Grizard J (1996) Phosphatidylinositol 3-kinase and p70 s6 kinase participate in the regulation of protein turnover in skeletal muscle by insulin and insulin-like growth factor I. Endocrinology 137:4087-4094

Deldicque L, Atherton P, Patel R, Theisen D, Nielens H, Rennie MJ, Francaux M (2008) Decrease in Akt/PKB signalling in human skeletal muscle by resistance exercise. Eur J Appl Physiol 104:57-65

Durieux AC, Bonnefoy R, Manissolle C, Freyssenet D (2002) Highefficiency gene electrotransfer into skeletal muscle: description and physiological applicability of a new pulse generator. Biochem Biophys Res Commun 296:443-450

Durieux AC, Bonnefoy R, Busso T, Freyssenet D (2004) In vivo gene electrotransfer into skeletal muscle: effects of plasmid DNA on the occurrence and extent of muscle damage. J Gene Med 6:809816

Durieux AC, Desplanches D, Freyssenet D, Fluck M (2007) Mechanotransduction in striated muscle via focal adhesion kinase. Biochem Soc Trans 35:1312-1313

Eliasson J, Elfegoun T, Nilsson J, Kohnke R, Ekblom B, Blomstrand E (2006) Maximal lengthening contractions increase p70 S6 kinase phosphorylation in human skeletal muscle in the absence of nutritional supply. Am J Physiol Endocrinol Metab 291:E1197-E1205

Fluck M, Hoppeler H (2003) Molecular basis of skeletal muscle plasticity-from gene to form and function. Rev Physiol Biochem Pharmacol 146:159-216

Fluck M, Waxham MN, Hamilton MT, Booth FW (2000) Skeletal muscle $\mathrm{Ca}(2+)$-independent kinase activity increases during either hypertrophy or running. J Appl Physiol 88:352-358

Fluck M, Ziemiecki A, Billeter R, Muntener M (2002) Fibre-type specific concentration of focal adhesion kinase at the sarcolemma: influence of fibre innervation and regeneration. J Exp Biol 205:2337-2348

Fluck M, Schmutz S, Wittwer M, Hoppeler H, Desplanches D (2005) Transcriptional reprogramming during reloading of atrophied rat soleus muscle. Am J Physiol Regul Integr Comp Physiol 289:R4R14

Fluck M, Mund SI, Schittny JC, Klossner S, Durieux AC, Giraud MN (2008) Mechano-regulated Tenascin-C orchestrates muscle repair. Proc Natl Acad Sci USA 105:13662-13667

Flück M, Carson JA, Gordon SE, Ziemiecki A, Booth FW (1999) Focal adhesion proteins FAK and paxillin increase in hypertrophied skeletal muscle. Am J Physiol 277:C152-C162

Fujita S, Abe T, Drummond MJ, Cadenas JG, Dreyer HC, Sato Y, Volpi E, Rasmussen BB (2007) Blood flow restriction during low-intensity resistance exercise increases $\mathrm{S} 6 \mathrm{~K} 1$ phosphorylation and muscle protein synthesis. J Appl Physiol 103:903-910

Gan B, Yoo Y, Guan JL (2006) Association of focal adhesion kinase with tuberous sclerosis complex 2 in the regulation of s6 kinase activation and cell growth. J Biol Chem 281:37321-37329

Gehl J, Sorensen TH, Nielsen K, Raskmark P, Nielsen SL, Skovsgaard T, Mir LM (1999) In vivo electroporation of skeletal muscle: threshold, efficacy and relation to electric field distribution. Biochim Biophys Acta 1428:233-240

Gingras AC, Raught B, Sonenberg N (2001) Regulation of translation initiation by FRAP/mTOR. Genes Dev 15:807-826

Gordon SE, Fluck M, Booth FW (2001) Selected contribution: skeletal muscle focal adhesion kinase, paxillin, and serum response factor are loading dependent. J Appl Physiol 90:1174-1183

Hemmings BA (1997) Akt signaling: linking membrane events to life and death decisions. Science 275:628-630

Hunter T (2000) Signaling-2000 and beyond. Cell 100:113-127

Ingber DE (2006) Cellular mechanotransduction: putting all the pieces together again. FASEB J 20:811-827

Isotani S, Hara $\mathrm{K}$, Tokunaga $\mathrm{C}$, Inoue $\mathrm{H}$, Avruch $\mathrm{J}$, Yonezawa $\mathrm{K}$ (1999) Immunopurified mammalian target of rapamycin 
phosphorylates and activates p70 S6 kinase alpha in vitro. J Biol Chem 274:34493-34498

Kimball SR, Horetsky RL, Jefferson LS (1998) Signal transduction pathways involved in the regulation of protein synthesis by insulin in L6 myoblasts. Am J Physiol 274:C221-C228

Kimball SR, O’Malley JP, Anthony JC, Crozier SJ, Jefferson LS (2004) Assessment of biomarkers of protein anabolism in skeletal muscle during the life span of the rat: sarcopenia despite elevated protein synthesis. Am J Physiol Endocrinol Metab 287:E772-E780

Laser M, Kasi VS, Hamawaki M, Cooper G, Kerr CM, Kuppuswamy D (1998) Differential activation of p70 and p85 S6 kinase isoforms during cardiac hypertrophy in the adult mammal. J Biol Chem 273:24610-24619

Malik RK, Parsons JT (1996) Integrin-dependent activation of the p70 ribosomal S6 kinase signaling pathway. J Biol Chem 271:2978529791

Nader GA, Esser KA (2001) Intracellular signaling specificity in skeletal muscle in response to different modes of exercise. J Appl Physiol 90:1936-1942

Napoli R, Gibson L, Hirshman MF, Boppart MD, Dufresne SD, Horton ES, Goodyear LJ (1998) Epinephrine and insulin stimulate different mitogen-activated protein kinase signaling pathways in rat skeletal muscle. Diabetes 47:1549-1554

Pallafacchina G, Calabria E, Serrano AL, Kalhovde JM, Schiaffino S (2002) A protein kinase B-dependent and rapamycin-sensitive pathway controls skeletal muscle growth but not fiber type specification. Proc Natl Acad Sci USA 99:9213-9218

Pullen N, Thomas G (1997) The modular phosphorylation and activation of p70s6k. FEBS Lett 410:78-82
Rennie MJ (2007) Exercise- and nutrient-controlled mechanisms involved in maintenance of the musculoskeletal mass. Biochem Soc Trans 35:1302-1305

Reynolds TH, Bodine SC, Lawrence JC Jr (2002) Control of Ser2448 phosphorylation in the mammalian target of rapamycin by insulin and skeletal muscle load. J Biol Chem 277:17657-17662

Romer LH, Birukov KG, Garcia JG (2006) Focal adhesions: paradigm for a signaling nexus. Circ Res 98:606-616

Sale EM, Atkinson PP, Arnott CH, Chad JE, Sale GJ (1999) Role of ERK1/ERK2 and p70S6K pathway in insulin signalling of protein synthesis. FEBS Lett 446:122-126

Samarel AM (2005) Costameres, focal adhesions, and cardiomyocyte mechanotransduction. Am J Physiol Heart Circ Physiol 289:H2291-H2301

Sandri M (2008) Signaling in muscle atrophy and hypertrophy. Physiology 23:160-170

Spangenburg EE, LeRoith D, Ward CW, Bodine S (2008) A functional insulin-like growth factor receptor is not necessary for loadinduced skeletal muscle hypertrophy. J Physiol 586:283-291

Terzis G, Georgiadis G, Stratakos G, Vogiatzis I, Kavouras S, Manta P, Mascher H, Blomstrand E (2008) Resistance exercise-induced increase in muscle mass correlates with p70S6 kinase phosphorylation in human subjects. Eur J Appl Physiol 102:145-152

Thomas G, Hall MN (1997) TOR signalling and control of cell growth. Curr Opin Cell Biol 9:782-787

Thomson DM, Gordon SE (2006) Impaired overload-induced muscle growth is associated with diminished translational signalling in aged rat fast-twitch skeletal muscle. J Physiol 574:291-305 